\title{
Mode de fonctionnement rituel et production musicale chez les Bororo du Mato Grosso
}

\section{Riccardo Canzio}

\section{OpenEdition}

\section{Journals}

Édition électronique

URL : http://journals.openedition.org/ethnomusicologie/2430

ISSN : 2235-7688

\section{Éditeur}

ADEM - Ateliers d'ethnomusicologie

Édition imprimée

Date de publication : 1 janvier 1992

Pagination : 71-95

ISBN : 978-2-8257-0456-1

ISSN : $1662-372 X$

\section{Référence électronique}

Riccardo Canzio, « Mode de fonctionnement rituel et production musicale chez les Bororo du Mato Grosso », Cahiers d'ethnomusicologie [En ligne], 5 | 1992, mis en ligne le 15 décembre 2011, consulté le 04 mai 2019. URL : http://journals.openedition.org/ethnomusicologie/2430 


\title{
MODE DE FONCTIONNEMENT RITUEL ET PRODUCTION MUSICALE CHEZ LES BORORO DU MATO GROSSO
}

\author{
Riccardo Canzio
}

\section{Les Bororo et leur situation actuelle ${ }^{1}$}

Dès les premiers contacts avec les Européens, les Bororo ont été l'objet de la curiosité des voyageurs qui nous ont laissé de nombreuses descriptions. Ils ont exercé une grande fascination sur des générations d'ethnologues qui ne les connaissaient qu'à travers une littérature bigarrée; la société bororo y apparaît comme un modèle d'organisation sociale dualiste caractérisé par la richesse de sa mythologie, la complexité de son univers symbolique et l'intensité de sa vie rituelle. Voyageurs, missionnaires et ethnologues les ont approché par le biais de leurs a priori; leurs écrits en témoignent. Dans le monde francophone, cette population est connue grâce aux travaux de Lévi-Strauss (notamment 1955 et 1958) qui a séjourné dans une communauté bororo au milieu des années trente et qui, pour l'élaboration de ses théories, a puisé largement dans ses observations personnelles.

Sous le poids d'une telle intellectualisation, la société bororo risque de devenir à nos yeux un mythe exemplaire correspondant à une réalité mal comprise. Ainsi, l'image de cette société prend souvent la forme qu'un groupe d'intérêt (ethnologues ou organismes internationaux d'aide, théologiens de la libération ou missionnaires conservateurs, grands propriétaires terriens ou paysans désherités à la recherche de terres) veut bien lui attribuer.

Il n'y a pas longtemps, on a prétendu que les Bororo n'existaient plus comme groupe ethnique, leur assimilation à la société brésilienne et leur incorporation aux couches les plus défavorisées de la société ayant été telles qu'on ne pouvait plus parler de culture bororo ni donc de Bororo. Certes, comme d'autres ethnies des basses terres du Brésil, ils furent dépossédés de leurs terres ancestrales et n'arrivèrent plus à vivre de la pêche, de la chasse et de la cueillette comme ils le faisaient autrefois. Ils survivaient à peine - et certains groupes le font encore -

Cet article repose sur nos observations de cette société et ses rituels. Nous avons eu l'occasion de séjourner plusieurs mois au village de Garças dans la réserve indigène de Meruri au Mato Grosso et d'assister au déroulement de cérémonies funéraires. L'élaboration d'une partie des matériaux collectés a déjà donné lieu à une publication (Canzio 1989) dont certains passages seront discutés ici. Nous remercions les habitants du village de Garças et en particulier Antonio Kanajó de Meruri ainsi que Gonçalo Ochoa et Mario Bordignon pour leur aide inappréciable pendant les différentes périodes de ma recherche. 
comme travailleurs ruraux toujours exploités et en porte-à-faux avec les valeurs de la société environnante. Cela entraîna une fraction importants de la population à décider collectivement, il y a une trentaine d'années déjà, de cesser de se reproduire, leur connaissance d'abortifs et de moyens contraceptifs naturels les aidant dans ce sens. Ce phénomène a été remarqué aussi chez d'autres ethnies de la région; un témoignage déchirant, recueilli dans les années soixante au village de Pojobari, illustre ce drame dans toute son intensité.

«Nous ne pouvons plus vivre comme des Bororo et nous ne vivrons pas comme des Brésiliens. Donc nous avons decidé de ne pas vivre du tout: nous mourrons à la façon bororo, un par un et après nous il n'y aura plus rien » (Crocker 1985: 330).

Aujourd'hui, cette tendance a été renversée et il existe environ huit cents hommes, femmes et enfants se disant Bororo, regroupés en quatre réserves indigènes au Mato Grosso. Certaines institutions, croyances et quelques aspects du mode de vie des Bororo n'ont plus les caractéristiques d'autrefois, mais un noyau irréductible de pratiques rituelles donnent encore un sens à la vie de nombreux individus. Le problème posé par l'acculturation est réel. Considérant le monde compliqué qui les environne, les Bororo, comme d'autres ethnies de cette aire culturelle, ont de plus en plus tendance à dévaloriser le leur. Partagés entre leur propre vision du monde et celle des Brésiliens, les Bororo finissent par vivre dans une sorte de crépuscule spirituel où très souvent règne le désespoir.

Cependant une vie rituelle relativement active, dont le chant constitue une activité indissociable, continue à exister chez certains groupes. Cette activité garde une signification capitale pour les gens qui la pratiquent, et nous constatons à travers sa réalisation une affirmation du sens communautaire. Ce processus constitue une sorte de rempart contre les assauts auxquels la société est soumise et qui ont fragilisé à l'extrême ses structures. Ces activités rituelles semblent aider certains groupes à reconstituer symboliquement le tissu de la communauté et à insuffler à leur culture une vitalité nouvelle.

L'importance primordiale de ces pratiques nous amène dans cet article à aborder la problématique que nous pose la description du phénomène musical dans les rites bororo. Notre thèse, ici, est que dans cette société, le phénomène musical peut être mieux compris à travers une description detaillée de la production musicale et du fonctionnement rituel. Par là nous entendons une tentative de description, d'interprétation et de contextualisation des actions rituelles et des interactions entre les individus donnant lieu à la production sonore, pour évaluer les limites dans lesquelles le produit musical des cérémonies bororo est formalisable.

En premier lieu, nous examinerons sommairement la société, les notions qui la soutiennent et la forme que prend la vie rituelle. Ensuite, nous discuterons les changements de conditions de cette société soumise aujourd'hui à de fortes pressions extérieures, ainsi que les réponses adaptatives qui transforment sa vie rituelle. Nous examinerons le type de problèmes que pose à l'ethnomusicologue la description du monde sonore des Bororo dans sa complexité où chant, texte, cosmologie, liens sociaux et structure parentale sont difficiles à démêler. Nous 
essaierons de déterminer ce qui est effectivement formalisable et ce qui ne l'est pas dans ce monde sonore. Nous proposerons ensuite une description sommaire du mode de production des chants préparatoires à une cérémonie de nomination.

Finalement, l'observation du mode de fonctionnement du chant, de la musique et des actions rituelles des cérémonies bororo dans la vie sociale nous amènera à des réflexions sur leur rôle comme composant constitutif de la construction symbolique de cette société, sur leur importance comme référent identitaire, ainsi que sur sa valeur comme stratégie de survie.

En annexe, une analyse formelle assez technique et méthodologiquement motivée propose au lecteur l'écoute guidée des passages choisis d'une publication discographique récente (Canzio 1989).

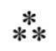

Deux siècles et demi après l'arrivée des premiers Portuguais au Brésil, un front de colonisation est entré en contact avec les Bororo. Au cœur du Mato Grosso, ce peuple était l'ethnie dominante d'une région s'étendant de la frontière bolivienne, à l'ouest, jusqu'au fleuve Araguaia au sud de l'actuel État de Goiás, mais il était concentré sur les rives des fleuves Cuiabá et São Lourenço. On en estimait la population, au début du $\mathrm{XIX}^{\mathrm{e}}$ siècle, à dix mille individus environ (Bordignon 1987:2)

Les premiers témoignages du contact sporadique des Bororo avec les bandeiras (expéditions composées de portuguais, de Noirs et de quelques Indiens et fer de lance de la pénétration coloniale dans l'intérieur inhospitalier du Brésil de l'époque), bien avant un contact suivi avec le front de colonisation, datent du milieu du XVIII ${ }^{\mathrm{e}}$ siècle. Malgré la durée du contact et l'intégration de certains groupes comme main-d'œuvre paysanne, les Bororo ont conservé, dans une large mesure, le modèle traditionnel de vie villageoise. Ce qui leur a donné une relative autonomie pour perpétuer des activités rituelles qui n'offrent aucun intérêt économique à la civilisation qui les entoure.

La coexistence entre la religion traditionnelle et le christianisme chez les Bororo habitant les missions et en contact continu avec la «civilisation", date des débuts du siècle, quand les pères Salésiens fondèrent les premières missions au Mato Grosso. Bien que l'attitude des missionnaires ait beaucoup changé avec l'avènement de la théologie de la libération dans les années soixante, il n'en demeure pas moins que le christianisme reste vu plutôt pour son aspect social et comme élément d'adaptation à la culture environnante que comme une religion avec un contenu théologique et des valeurs auxquelles on adhère.

Toute référence actuelle à leur culture de la part des Bororo fait allusion au passé, à l'époque où il y avait des grands chasseurs et une pêche abondante, des hommes forts et respectueux des prescriptions d'ordre alimentaire et rituel. Tout cela est symptomatique d'un repli sur les valeurs établies comme dernier refuge face aux avances d'une société environnante qui les condamne inexorablement à l'assimilation. 


\section{La société}

L'intégralité de la société traditionnelle bororo est fondée sur l'observance rigoureuse d'un code de réciprocité très élaboré et sur un régime de droits et de devoirs entre les clans matrilinéaires des deux moitiés exogames. La structure dualiste de la société se reflète dans la topographie du village; celui-ci, de forme circulaire, est divisé par un axe est-ouest délimitant spatialement deux moitiés.

Une case située au centre du village est le lieu de rassemblement des hommes; les femmes y sont invitées lors de certaines cérémonies pour former le chœur qui soutient et paraphrase le chant des hommes. Dans la cour cérémonielle (bororo) située à l'ouest de cette case, se déroulent la plupart des cérémonies.

Chaque clan possède son ensemble d'ornements, de peintures corporelles, de noms personnels, de chants, de mythes et de privilèges. Entre les clans existe un système d'obligations réciproques qui est le résultat d'échanges et de transactions bien établies, la plus importante d'entre elles étant la réalisation des cérémonies funéraires pour un défunt d'un clan de la moitié opposée.

La mort est considérée par les Bororo comme une atteinte à l'équilibre existant entre la société et la nature; quand un Bororo meurt, toute la société est symboliquement menacée. Les forces hostiles de la nature sont tenues pour responsables de cette mort; le dommage doit être réparé et l'équilibre retrouvé. Le rite funéraire, mise en scène du monde symbolique, est réalisé pour aider l'âme du défunt à s'intégrer à l'âme collective (aroe) des ancêtres.

Les Bororo pratiquent une double inhumation. Le corps sera d'abord déposé dans une fosse temporaire jusqu'à ce que les chairs soient putréfiées. La fosse est arrosée régulièremet et l'on vérifie de temps à autre l'état de décomposition. Quelques semaines plus tard aura lieu la phase finale des funérailles; les ossements seront lavés, peints, ornés de plumes, puis places dans un panier spécialement tissé et décoré. Celui-ci sera déposé à son tour dans des grottes ou dans le fond des mares qui abondent dans la région. Au cours des semaines qui séparent les deux enterrements, une série de jeux-représentations seront organisés. Grâce à des peintures corporelles et à des ornements (plumes, dents de fauves, becs d'oiseaux) on met en scène des événements mythiques. Toute cérémonie chez les Bororo a pour but la répresentation de l'aroe, mais un clan ne représente jamais son propre aroe, c'est un clan de la moitié opposée qui s'en charge.

Il existe deux principes cosmologiques chez les Bororo autour desquels s'organise toute la pensée traditionnelle: aroe et bope. L'aroe inclut les ancêtres et les héros culturels ainsi que tout ce qui est nommable, nature ou artefact, réel ou imaginaire. L'aroe est donc l'essence de tout concept ou entité: animal, poisson, plante, emblème clanique, astre, chant ou instrument. Il est lié à la structure parentale de la société et à la configuration topographique du village. A chaque clan appartient une des huit catégories de l'aroe. Les bope sont des manifestations de la nature et sont à l'origine des transformations organiques: naissance, croissance, maladie et mort; ils sont associés à des phénomènes et processus naturels - l'éclair, le tonnerre, la pluie, la chaleur, le froid - et à certains animaux dans lesquels s'incarne le chamane des esprits (bari) s'incarne. Il y a des bope agissant pour le 


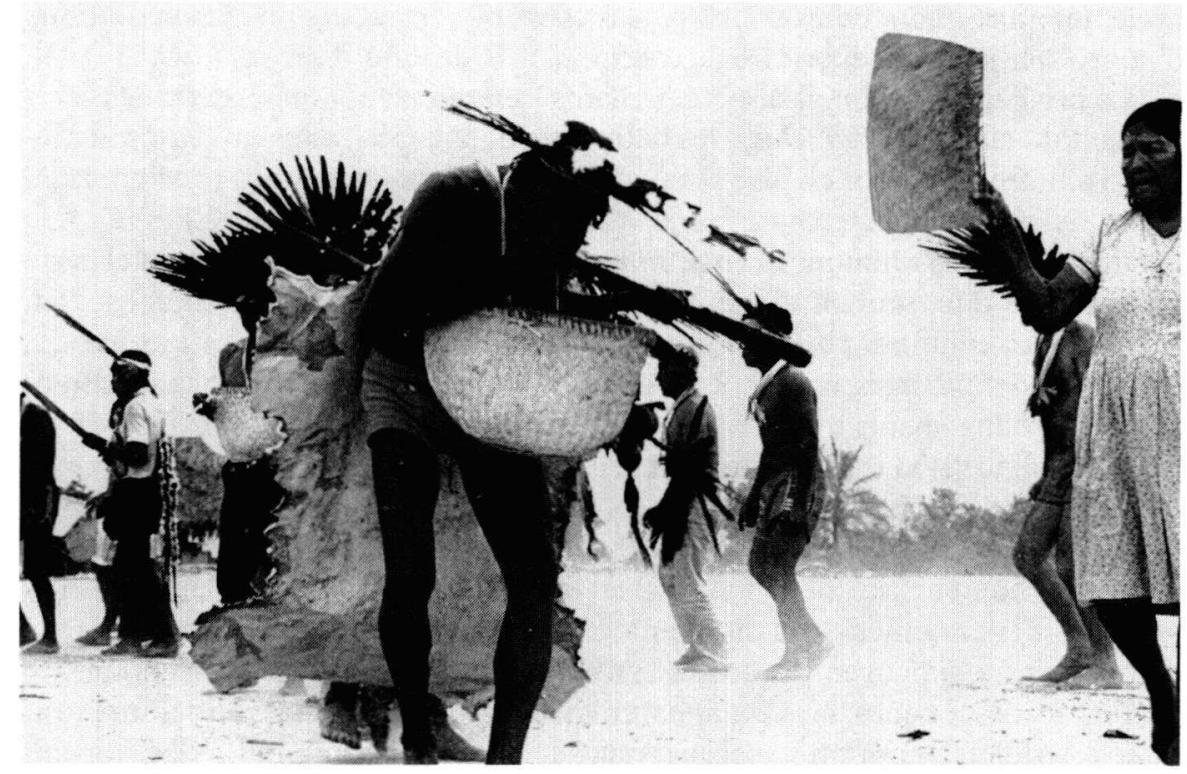

Fig. 1: Le chamane des âmes mène la procession dansante en jouant de l'ika et en portant autour de son cou le panier qui contiendra les ossements du mort.

Photo: Priscilla Ellis.

bien des hommes et d'autres qui leur portent préjudice; ces derniers se manifestent lorsque la société manque au code qui gouverne ses relations avec la nature.

Ces deux concepts ont été décrits (entre autres) par Crocker (1985); nous discuterons plus loin de leur applicabilité à une éventuelle description musicologique du rituel. Récemment, Viertler (1991:123), dans un excellent essai d'interprétation des catégories autochtones bororo, a associé l'aroe aux facultés mentales supérieures conscientes telles que la langue, la mémoire, la sociabilité, le travail et les activitiés rituelles, et les bope aux phénomènes psychiques inconscients ou subconscients liés aux rêves, prémonitions, passions et désirs non contrôlés, ainsi qu'à des éléments qui interfèrent avec toute forme de cooperation humaine.

\section{Les chants}

Les textes ont été classés (Colbacchini 1925:120, classification reprise par Albisetti et Venturelli 1976) en chants funéraires et chants préliminaires aux expéditions collectives de chasse et de pêche. Cette classification ne correspond pas à une catégorie autochtone explicite, même si elle reflète une distinction liée à certaines caractéristiques de la réalisation musicale (seuls certains individus peuvent être meneurs dans les cérémonies préliminaires, une seule paire de petits hochets y est utilisée, ce sont les seules cérémonies réalisées en dehors du 
cycle funéraire, etc.). Pourtant, cette classification représente une façon pratique d'approcher le vaste répertoire de cérémonies et des rites bororo.

Le privilège de l'organisation des chants funéraires fut donné aux chefs cérémoniels du clan des badojeba, "les bâtisseurs du village», dans un temps mythique. Ils furent les héros civilisateurs qui humanisèrent les Bororo par l'intermédiaire de pratiques cérémonielles jusqu' alors inconnues. Aujourd'hui, c'est le cercle des "grands hommes" (boe eimejera) qui choisit, en fonction du clan du défunt et du représentant du défunt ou de la personne pour qui le rituel se fait, selon le cas, celui qui chantera quoi et à quel moment. L'ordre et la suite des chants ne sont pas figés, mais ils le seront à l'occasion de la réalisation des cérémonies. Ces changements n'altèrent pas l'ordre rituel des funérailles, mais il existe une variabilité selon le statut social et le prestige du mort dans la suite des chants.

Chaque clan garde jalousement ses droits de représentation ainsi que ses droits d'exécution des chants claniques, mais ceux-ci peuvent être délégués. Le choix des chanteurs se fait en fonction de leurs connaissances et de leur capacité, même si la prérogative de la distribution des chants revient aux «propriétaires» de ces chants qui peuvent, et de fait le font, déléguer leur droits d'exécution.

Le rôle des femmes, bien qu'apparemment secondaire dans la structure musicale, est d'une importance sociale cruciale. Selon la coutume, elles doivent s'asseoir derrière leur mari et l'accompagner, seules ou en chœur, quand il chante; elles doivent aussi pleurer au moment où les chants évoquent un défunt ou des morts d'autrefois. L'aroe étant toujours évoqué pendant les cérémonies, les «âmes» des morts récents ainsi que celles des ancêtres se rencontrent à l'occasion des funérailles. Tout homme qui se respecte sera toujours accompagné dans le chant par sa femme ou par une parente clanique.

L'apprentissage des chants ne se fait pas d'une façon formelle; ils sont appris durant la célébration des cérémonies auxquelles, en principe, assistent tous les membres de la communauté aussi bien que des non résidents du village tels que les affiliés claniques du mort et des individus d'un certain prestige spécialement invités pour la circonstance. L'occasion d'écouter maintes fois dans une vie les textes des chants, associée à la remarquable mémoire pour laquelle les Bororo sont connus, contribuent à la transmission et à la préservation des textes. Des caractéristiques de la performance qui veulent que dans certains types de chant le texte soit dit à haute voix avant son exécution, le caractère répétitif de la plupart des chants comportant un élément variable de substitution et le rôle du meneur comme régisseur et organisateur du rite sont des facteurs qui favorisent fortement l'apprentissage, non seulement des textes, mais aussi des rythmes fournis par l'accompagnement des hochets.

Les textes évoquent des expéditions collectives de chasse et de pêche et représentent une codification complexe des techniques liées aux activités traditionnelles de subsistance et aux connaissances spécifiques de la flore et de la faune du vaste territoire ancestral. Les âmes des ancêtres sont suppliées d'entrâ̂ner le gibier dans les pièges et d'assurer une bonne chasse. 


\section{Faire et parfaire}

Nous trouvons que chez les Bororo, la notion de faire rituellement est à la base des conceptions «musicales». En effet, les Bororo ne connaissant aucun terme pour nommer la musique, le mot roia, signifiant «façon de faire, action» (Albisetti et Venturelli 1962:908) désigne l'action rituelle et le chant luimême ${ }^{2}$. Il y a un faire rituel et une recherche de la perfection dans ce faire. Cette recherche de la perfection est aussi une caractéristique d'autres aspects de la vie traditionnelle bororo; adhésion stricte aux prescriptions dans la confection d'emblèmes claniques ou dans la réalisation de peintures corporelles, justesse de comportement social, respect des restrictions alimentaires, etc. Mais avant tout, la précision dans l'action rituelle est de rigueur.

Certes, les conditions de vie actuelles imposent des contraintes d'ordre matériel à la réalisation de certaines cérémonies. Un compromis sur la forme rituelle s'avère souvent indispensable, mais une fois établies les conditions dans lesquelles le rite se déroulera, elles seront respectées à la lettre. Les changements observés semblent être dus à divers facteurs tels que l'impossibilité de se procurer les matériaux nécessaires à la confection des objets rituels ou l'absence de représentants de certains clans dans un village au moment du rite, ce qui implique une modification des textes et de l'ordre des cérémonies.

Certains types de changements circonstanciels, surtout au niveau des actions rituelles, et même des changements ou omissions textuels sont admis facilement, tandis que rien n'est altérable dans ce qui touche à l'aroe. On a même pu constater des instances de réorganisation de la parenté, par le transfert de certains individus d'un clan à un autre, afin de ré-équilibrer la structure de la société par rapport à l'idéal proposé par l'aroe. Cela nous donne une idée de l'ampleur que ce principe charnière prend chez les Bororo et de la mesure de la nécessité ressentie par eux de maintenir vivant l'essentiel des schémas mentaux qui les guident.

Ainsi nous constatons que d'un côté il s'opère des modifications sur la forme des rites, mais que de l'autre, la rigidité de certaines conditions empèche que les changements dépassent certaines bornes. Il existe un aspect paradoxal entre, d'une part, certains éléments de prescription rituelle absolus et donc non modifiables - cette attitude du tout ou rien nécessaire à l'existence d'un rituel et d'autre part, les changements d'ordre formel que subissent les cérémonies, qui sont plus ou moins facilement acceptés. En faisant la distinction entre le contexte circonstanciel immédiat dans lequel une cérémonie se déroule et le contexte culturel au sens large (c'est-à-dire croyances, mythes, structure sociale), cet aspect paradoxal peut s'expliquer plus facilement.

Par exemple, en ce qui concerne le dépôt final du panier contenant les ossements du défunt, un compromis a été trouvé entre la vie cérémonielle tradition-

On trouve aussi le mot akodo, «produire un son» (Albisetti et Venturelli, 1962:46), mais il connote le fait physique de la production sonore et non l'organisation des sons. 
nelle et un enterrement chrétien. Le panier était deposé dans des caves ou dans le fond de mares; aujourd'hui il est souvent enterré dans un tombeau dans le cimetière de la mission voisine. Une fois le rite accompli, les circonstances du dépôt final des ossements ne semblent pas être d'une importance majeure. Mais la cérémonie de rachat (mori) réalisée quand un Bororo est blessé par un élément naturel ou tué, et qui consiste, de la part du «substitut» ou représentant vivant du mort, à abattre une bête sauvage comme rançon demandée à la nature en compensation de la mort et à offrir sa peau à un parent du défunt, ne peut avoir lieu que si l'on a la possibilité effective de tuer un tel animal (ou de se procurer, par don ou achat, une peau). Ce rite est aujourd'hui de plus en plus rare, ce qui empêche l'accomplissement d'échanges importants pour les individus et retarde le rétablissement des liens de réciprocité essentiels à une vie communautaire harmonieuse. Mais le mori est un principe du système de réciprocité qui reste immuable, et souvent, l'accomplissement des obligations qu'il implique est réalisé de longues années après les funérailles.

Ainsi, le faire rituel permet des changements et un certain degré d'adaptation aux conditions changeantes de l'environnement social et écologique; tandis que le parfaire dicté par les conditions restrictives de l'aroe ne permet pas de variation ou de substitution. Si l'intégrité de l'aroe peut être assurée, le système de pensée symbolique peut continuer à être investi de sens et de signification malgré les atteintes aux limites structurelles des relations sociales traditionnelles de la communauté.

On peut voir comment les prescriptions de la performance fonctionnent dans un double mouvement de va-et-vient. Elles décrivent, parce que leur intention est précisément de guider la réalisation des actions rituelles dans toutes sa minutie et en même temps, elles font exister le système cosmologique dans sa complexité. Ce processus, devenu classique dans la description des rites, a été déjà noté par Geertz et exprimé clairement quand il remarque qu'un système de pensée est à la fois un modèle pour et un modèle de la société:

«Ici, la théorie est un modèle que guide la façon dont les relations physiques sont organisées: c'est un modèle pour la 'réalite'. En ce qui concerne les systèmes psychologiques et sociaux et aussi pour des modèles culturels qu'on ne pourrait pas appeler 'théories' mais plutôt 'doctrines', 'mélodies' ou 'rites', le cas n'est pas différent. A l'opposé des gènes et d'autres sources d'information non-symboliques qui sont seulement des modèles pour et non des modèles de, les traits culturels (cultural patterns) ont un double aspect intrinsèque: ils donnent de la signification - c'est-à-direune forme conceptuelle objective - à la réalité sociale et psychologique, tout en la formant et en se conformant à elle à la fois». (Geertz 1973: 93) 


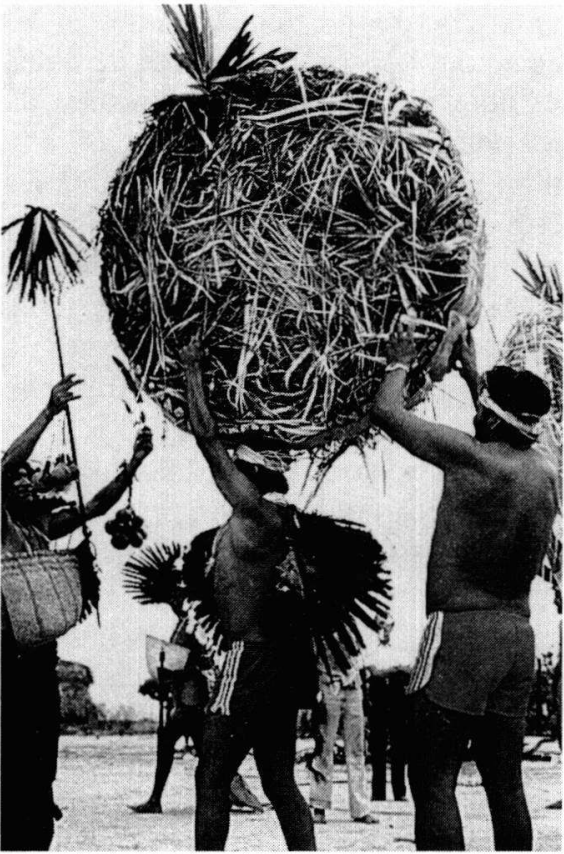

Fig. 2: Le jeu du marido, une des représentations mythologiques ayant lieu juste avant l'enterrement définitif.
Photo: Priscilla Ellis.

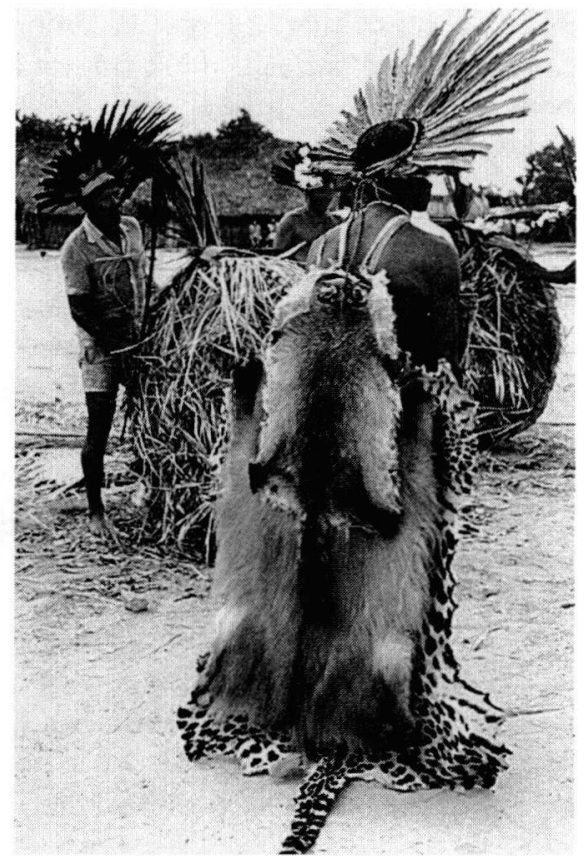

Fig. 3: Peaux de bêtes obtenues comme mori, montrées à l'occasion du jeu du marido.

Photo: Priscilla Ellis.

\section{Le monde sonore}

La notion de monde sonore, plus ample que la notion de musique, nous donne la latitude nécessaire pour mieux comprendre et décrire la relation son/fonctionnement rituel ${ }^{3}$. Le concept de «musique» ne paraît pas à même d'englober tous les phénomènes sonores observables dans la société bororo. Les plaintes rituelles avec leur langage hautement codifié sont-elles des chants? Les trompes traversières $i k a$, symbolisant le héros culturel Bakororo et dont l'utilisation rituelle correcte semble plus importante que la bonne interprétation des appels correspondants, sont-elles des instruments de musique, des ustensiles cérémoniels ou des «concepts»? Les appels caractéristiques du powari, instrument censé répresenter l'aroe d'un mort, lancés lors des expéditions de chasse ou de pêche et certaines répresentations collectives de l'aroe, sont-ils de la "musique»? L'instrument même est-il un objet ou un signe? Un instrument comme n'importe quel

Le terme, mais non le concept de monde sonore, tel qu'il est utilisé ici est emprunté à Aytai (1985). 
autre «objet culturel» doit être compris comme un rapport entre les objets, les concepts et les percepts de la culture en question. Un certain nombre de questions de ce type se posent, pour lesquelles nous n'avons pas pour l'instant de réponse satisfaisante à donner; elles doivent pourtant être formulées.

D'ailleurs, et conformément à ce qu'on pourrait attendre, de multiples aspects de ce monde sonore ne pourront être expliqués que comme des épiphénomènes du contexte rituel. Par là je veux dire que certaines parties du résultat audible de l'ensemble des rites bororo sont la conséquence directe du système d'interaction sociale et du fonctionnement rituel. Par contre, d'autres parties de cet ensemble, tels que les chants, se prêteront à une analyse musicale formelle plus ou moins conventionnelle.

Dans la plupart des cas, l'ethnographe musical est contraint d'isoler la musique de son contexte pour pouvoir y appliquer les techniques de description et les modes d'analyse connus. On replacera le tout dans son contexte originel et on essaiera de voir quels paramètres du contexte agissent sur le système musical même. Nous constatons que, chez les Bororo, tout ce qui sonne est loin d'être analysable musicalement, que certains phénomènes sonores sont le produit de ce contexte et ne sont pas susceptibles d'une formalisation rigoureuse. Précisément parce que notre but est de décrire le phénomène «musical» dans sa totalité, nous ne pouvons négliger les particularités très spécifiques du contexte dans lequel la musique a lieu. Nous isolons et analysons le système musical dans la culture mais affirmons que, pour ne pas réduire l'univers musical de cette société à ce qui est seulement formalisable, ou à l'opposé, laisser de larges parties de ce monde sans description adéquate, cette approche d'ensemble s'avère inappréciable.

En effet, durant toute cérémonie, on entend une multiplicité de sons, résultant de la simultanéité des événements. Le phénomène (qui fait quoi à quel moment) n'est descriptible que comme une activité créative collective: l'assemblée des hommes chante accompagnée du chœur des femmes, on entend les plaintes rituelles des parents du défunt, quelqu'un souffle dans un powari pour appeler les aroe des ancêtres. Ce tout n'est explicable que par une description du contexte. En revanche, le chant lui-même, les manipulations textuelles et l'accompagnement instrumental sont, quant à eux, formalisables.

Si une musique est un système de relations, cette approche, qui aborde le monde sonore et l'examen des modes subtils d'interaction entre les acteurs du rituel qui le produisent, pourra nous aider à fournir une description plus complète du phénomène examiné. En même temps nous nous défendons d'appeler musique tout ce monde sonore, malgré la signification et la valeur esthétique que pourrait lui attribuer un observateur extérieur. Par musique nous entendons un acte créatif volontaire d'organisation des sons; une combinaison fortuite de sons, si agréable et acceptable (et acceptée) fût-elle ne nous parait pas remplir ces conditions. Seeger va dans notre sens quand il dit que:

«La musique est beaucoup plus que les sons enregistrés. La musique est l'intention de faire quelque chose qu'on appelle musique (ou structurée comme ce que nous appelons musique) et différent d'autres types de son » (Seeger 1987: xiv). 
La description du phénomème musical lui-même, qui est aussi du ressort de l'ethnomusicologue, est une autre question parce que chaque source sonore est susceptible d'être décrite, identifiée, expliquée, et le résultat audible également compris même s'il n'est pas voulu ou organisé.

Confrontés à un système sonore dont nous ne connaissons pas les règles, il est important dans notre description de ne pas le juger sur les simples apparences sonores, mais précisément d'essayer de définir ces règles qui vont le déterminer. Les aspects d'interaction entre les individus durant la performance nous aident à percevoir les différences entre le résultat sonore et ce qui devrait être: le normatif et le réellement écouté. Ainsi nous arrivons à voir quelles sont les forces et la pensée qui génèrent ce que nous écoutons.

Les systèmes de description classiques utilisés par l'ethnomusicologie ne suffisent pas à fournir une explication satisfaisante d'un complexe sonore comme celui auquel nous sommes confrontés chez les Bororo. Pour interpréter ce monde sonore, nous formalisons le normatif et décrivons sa réalisation, tout en considérant les faits de performance qu'expliquent les déviations du normatif. Autrement dit, nous formalisons une structure, le système abstrait de règles de pensée, puis nous décrivons les événements particuliers qui ont lieu lors de l'application de ces règles. La description de ces événements nous renvoie à notre système de règles et le phénomène en question est reinterprété en fonction de l'usage fait de ces régles.

La notion de monde sonore, plus qu'une méthode d'analyse, est un nouveau paradigme (au sens kuhnien) qui propose une attitude heuristique envers l'analyse. Pour la compréhension du phénomène sonore en question, nous fonctionnons à deux niveaux différents et nous ne pouvons prévoir à l'avance à quel niveau d'analyse une structure audible pour être décrite: au niveau du noyau sonore formalisable (texte, métrique, rythmes, contours mélodiques, etc.) ou au niveau où l'ensemble de forces agissant à un moment determiné est analysable. Nous sommes redirigés d'un niveau à l'autre par les particularités des interactions sociales et des caractéristiques du phénomène lui-même, bien que le résultat sonore soit aléatoire.

Il y a donc un jeu entre les différents niveaux d'analyse: la signification de certains faits ne s'éclaircit que par une investigation fine du contexte, et les résultats que l'on en tire nous permettent de circonscrire les limites dans lesquelles notre analyse formelle est réalisable. Ainsi, la notion de forme dans ce monde sonore possède deux facettes: l'une «syntactique», détectable par une méthodologie d'analyse courante ou par des techniques de découverte ad hoc, et l'autre «sémantique», qui ne l'est pas par ces moyens, mais dont la signification se dégage d'un examen de la production sonore.

Si l'ethnomusicologie, telle qu'elle se présente actuellement, est une collection hétérogène de techniques adaptées à la diversité du comportement musical humain, pourquoi ne procéderait-elle pas selon ce paradigme qui représente, en effet, une position théorique ouverte, englobant tout l'audible mais n'excluant (par manque de méthodes de description ou par une attitude analitique réductive classique) ni la complexité de l'interaction entre les producteurs d'un phénomène sonore, ni aucun élément de sa perception? 


\section{Modes de production musicale et interaction sociale}

La parfaite structure dualiste des concepts cosmologiques qui imprègnent la société et ses institutions nous pousse à chercher d'autres structures à caractère binaire qui expliqueraient la totalité du phénomène musical selon les mêmes lignes de pensée. C'est la position adoptée par Seeger (1980: 7-42) qui nous montre, que pour le genre vocal akia des Suyá du Xingú, il existe un parallèle avec la dualité de certaines structures sociales. Ainsi y a-t-il dans la pensée traditionnelle bororo une série de complémentarités telles que, par exemple, l'opposition boe/barege, «homme/animal», dont le second terme inclut tout être vivant y compris d'autres groupes indigènes et la paire boe/baraedo, «indien/civilisé». Cette dernière notion est partagée avec la plupart des ethnies indiennes du Brésil et son apparition est le résultat du contact, lorsque l'«autre», le «civilisé» impose sa signification dans la pensée indigène et s'intègre à son monde conceptuel par la force de ses moyens technologiques et par sa capacité de contrôler l'environnement.

De même, selon Crocker (1985: passim), la société bororo s'articule encore autour d'une autre complémentarité: la paire bope/aroe; ses arguments sont convaincants et la formulation de cette opposition sert fort bien à éclairer nombre de phénomènes sociaux. Pourtant les pratiques rituelles significatives sur le plan sonore sont celles dont l'officiant est l'aroe etawaare, le chamane des âmes. En effet, c'est l'aroe auquel on fait appel pendant les cérémonies funéraires et les rituels préparatoires aux expéditions de chasse et de pêche. Tout ce qui conditionne le monde sonore des Bororo a affaire à l'aroe. Si dualité il y a dans ce domaine, elle ne s'exprime pas au niveau aroe/bope, comme le suggère Crocker pour d'autres aspects de la société. Elle oppose, de préference, le monde des vivants et le surnaturel: boe/aroe. L'existence de concepts tels que aroe ekeroia, "chants des âmes" et boe ekeroia, "chants de Bororo" semble suggérer une voie d'interprétation menant dans cette direction. D'un autre côté, l'existence de cette complémentarité conceptuelle est loin, à notre avis, d'expliquer la totalité du phénomène sonore chez les Bororo en termes d'oppositions binaires. C'est pour cela, donc, que nous tenterons ici, à travers une brève decription de la performance et dans l'annexe d'une façon plus détaillée, d'entrevoir quels types d'interactions ont lieu entre les participants durant une performance.

Pour ce faire, nous avons choisi l'oieigo, un chant propre au clan des geais (iwagududoge) associé aux cérémonies de nomination et aux rites préparatoires, aux expéditions collectives de chasse et de pêche et tout particulièrement à la chasse rituelle qui suit un décès. Il est interprété aussi lorsque quelqu'un s'est tiré d'une situation dangereuse. Dans la tradition mythologique des Bororo, nous trouvons l'histoire de Meriri Okwoda, un héros culturel du clan des singes (paiwo), qui fut à l'origine de cette cérémonie. Un jour, il pêcha un poisson d'une espèce inconnue, l'amena à sa hutte et se mit à le cuire dans un grand pot de terre. Lorsque l'eau commença à bouillir, une voix en sortit disant: «o-ie o-ie igo i-go »; il interpréta ces mots comme signifiant que le poisson disait être un animal totémique de son clan. C'était là un avertissement: il s'agissait d'un animal qu'il ne failait pas consommer avant que le chamane des esprits (bari) ne l'ait 


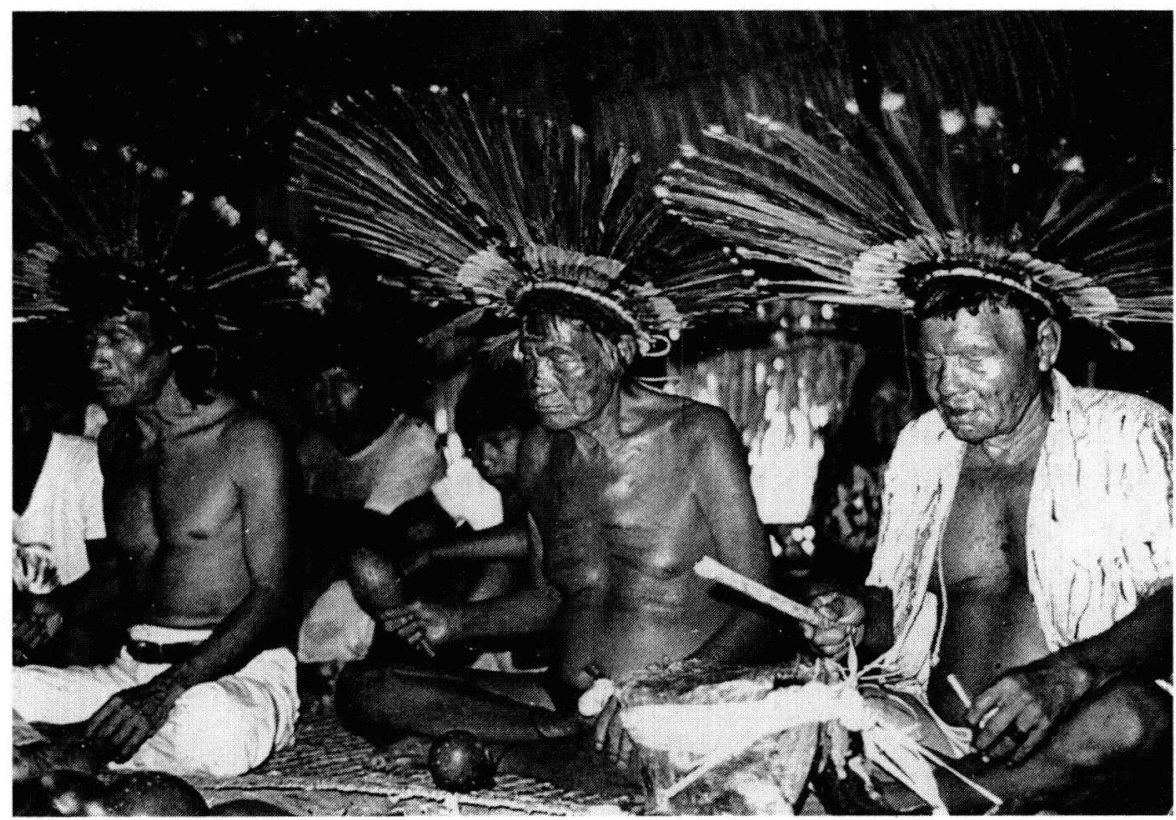

Fig. 4: Le chant assis nocturne. Le chœur des femmes au dernier plan.

Photo: Priscilla Ellis.

soumis à un rite de purification. La consommation d'un tel aliment pouvant entraîner la mort, les Bororo se servent de ce chant pour manifester leur joie d'avoir évité un danger (Albisetti et Venturelli 1976: 276).

L'exécution complète des préparatifs pour la cérémonie qui se déroulera le lendemain matin dure toute la nuit. Les strophes du oieigo, qui sont en prose, sont alternées avec des chants de nomination. Les deux premières strophes de l'oieigo sont d'abord récitées trois fois de suite ( $c f$. Canzio 1989: plage 9, index 1 et 2). Elles sont ensuite reprises en chantant (idem: index 3 et 4). Les deux strophes suivantes de l'oieigo sont à leur tour récitées (idem: index 5 et 6). Les chants de nomination sont alors entonnés (idem: index 7 à 16) puis les troisième et quatrième strophes sont chantées (idem: index 17 et 18).

Le texte de l'oieigo suit un canon fixe et des variations textuelles apparaissent selon le clan de celui qui offre et de celui qui reçoit l'oieigo. Il constitue une sorte de «grille» sur laquelle sont greffées les variations textuelles qui portent sur les emblèmes claniques et les noms propres des héros mythologiques. Le texte en Bororo des quatre premières strophes de l'oieigo est présenté dans l'annexe; chaque partie sera répétée à trois reprises. L'objet de cette répétition est de rappeler à l'assemblée des hommes le texte qui va être chanté par la suite. On pourra remarquer que le récitant consulte les chefs réunis pour pallier ses doutes et oublis concernant la structure du texte. Par exemple, à l'occasion, il demande le nom du héros qui doit figurer dans le texte chanté (index 2/temps 01:41). 
Une fois le texte du oieigo fixé, il fera partie du répertoire habituel et sera utilisé à des occasions variées pour évoquer la mémoire d'un défunt. Ici, il s'agit d'un «vieil» oieigo (une cérémonie déjà instaurée mais reprise pour l'occasion) chanté dans le contexte des préliminaires à une cérémonie de nomination (roia kidoguru paru $)^{4}$.

L'oieigo peut se faire avec les cérémonies de préparation à des expéditions de pêche, de chasse ou à certains jeux-représentations. Quand un «vieil» oieigo est chanté, son choix est le privilège celui qui organise l'expédition de pêche (par exemple) dans le but de nourrir toute la communauté, et c'est lui qui décidera à qui il sera dédié.

Les paroles des strophes de notre exemple enregistré font référence aux actions rituelles qui ont lieu le jour de l'instauration du oieigo. Les voici:

[1] Ici je propose le chant oieigo. Moi Bokodori, j'introduis ce chant pour commencer l'imposition de la ceinture-hochet. Ici il reste.

[2] Regarde ici, nous t'appelons. Notre diadème de plumes rouges de la queue d'un perroquet arara va être laissé au bord du village où tu arriveras. Regarde ici.

[3] Prenez-le. Quand vous le prendrez, attachez à sa tête le diadème orné de duvet qui se trouve dans la cour où les hommes se réunissent.

[4] Le son des hochets se fait entendre. Le brave vengeur Ki Bakororo amène la peau de l'ocelot.

La récitation commence; le meneur qui joue des petits hochets (bapo rogu) est debout près du poteau central de la maison des hommes; il regarde vers l'ouest, le lieu du héros Bakororo, et il est entouré de quelques hommes et des jeunes déjà initiés; d'autres le rejoignent au fur et à mesure. Peu avant, il a été convié par un des jeunes qui est allé le chercher chez lui et l'a amené en le tenant sa main, indiquant ainsi une invitation formelle à conduire les chants préparatoires. Seuls certains hommes peuvent être meneurs dans ce type de cérémonie et ont le droit de jouer des petits hochets. Ils doivent être formellement initiés, mémoriser un vaste répertoire et être toujours prêts à l'éxecuter. En effet, être meneur implique des obligations très lourdes envers la communauté; de là la réticence souvent manifestée à présent par les jeunes à assumer un tel rôle.

En écoutant le chant, les femmes se joignent progressivement au chœur accompagnées des jeunes enfants; elles s'asseyent par terre sur des nattes tout au long de la paroi est de la maison centrale.

La fonction du meneur est de conduire la performance, de rappeler les textes qui seront chantés et éventuellement de corriger des fautes d'interprétation. Mais il est important aussi qu'il arrive à créer l'atmosphère nécessaire à l'invocation des âmes. Il le fait avec des cris qui imitent les voix des aroe des différents animaux et parfois avec un accompagnement instrumental. On entend alors un

4 La notice du disque mentionne de façon erronée qu'il est chanté à l'occasion des préparatifs d'une expédition de chasse collective. 
roulement continu de hochets (à partir de l'index 7/ temps 10:27); c'est ce que les Bororo appellent "élever» le rythme, un procédé stylistique utilisé pour encourager les chanteurs. Chaque exécution collective est précédée d'une exposition préalable du modèle formel, musical et textuel, qui va être immédiatement chanté. Des sections formelles du texte sont marquées par des traits de percussion particuliers (voir par exemple la transition entre les sections 1 et 2 du oieigo, plage $9 /$ temps $4: 05)$. Un autre trait rythmique indique la fin d'un chant ou la fin de l'exposition du modèle formel (voir par exemple la plage 9, index 7/temps $8: 21$ ); après cela, le hochet gauche continue à battre la pulsation, indiquant qu'un autre chant va suivre immédiatement, et pour ne pas interrompre la continuité du son.

La connaissance de critères de réalisation de ce type et des rapports entre meneur et assemblée des hommes, le rôle des femmes, les actions correctives appliquées, etc. (voir détails dans l'annexe) nous permet d'avoir les éléments de jugement nécessaires pour nous situer à l'intérieur des canons esthétiques de la culture, nous permettant d'apprécier pleinement le complexe musical qui est en train de se dérouler.

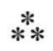

\section{Chant, mort, identité et survie}

Ce jour-là comme tant d'autres, la routine sans événements typiques de la vie quotidienne d'un village bororo prédominait, mais à l'annonce de la mort de cette vieille femme (on s'y attendait depuis quelque temps), le premier cri de deuil se fit entendre, puis d'autres s'y ajoutèrent ${ }^{5}$. Un chef de clan prit le gros hochets et se mit à entonner le grand chant solennel, le roia kurireu. Vite, les hommes le rejoignirent, les femmes arrivèrent peu à peu, s'assirent derrière eux, puis le chœur se forma. Alors tout bascula, l'élan de ce chant primordial semblait emmener de force la communauté entière après lui, dissipant l'apparent manque d'entrain régnant, et amorçant la longue série de cérémonies, rites et représentations mythologiques qui allaient se dérouler durant les semaines qui suivirent. Ils constituent le point central de la culture et de la vie rituelle bororo, en permettant aux Bororo de renouer les liens sociaux existant entre eux et de rétablir des rapports avec l'univers qui est le leur.

On affirma avec fierté que maintenant, on allait voir de vraies choses bororo. Effectivement, la cérémonie du premier enterrement précéda les représentations des aroe qui allaient se dérouler assez régulièrement pendant les deux mois suivants, en attendant le grand rituel final marquant l'enterrement définitif. Ainsi,

Voir des exemples de cris de deuil, mais dans un contexte différent, in Canzio (1989: fin plage 8/temps 9:33). 
par une sorte de paradoxe sinistre, la vitalité du monde bororo qui s'épuise irrévocablement ne retrouve sa force que grâce à la mort.

Il y a un clivage insurmontable entre cette vision du monde traditionnel et la réalité qui entoure les Bororo. L'univers intellectuel bororo, cette magnifique construction symbolique supportée par une riche mythologie et une cosmologie élaborée, était admirablement adapté au milieu écologique traditionnel, alors que les contacts intertribaux et les échanges avec le front de colonisation se déroulaient à une échelle de temps assez lente. Lors des premiers contacts, les Bororo se montrèrent curieux à l'égard du nouveau monde qui s'ouvrait devant eux. Ils développèrent leur système traditionnel d'interprétation de l'univers, c'est-à-dire l'attribution de tout élément de cognition à une des parties de l'aroe, pour permettre l'assimilation - qui se fit en douceur au début - des objets et des concepts issus de ce contact. Ils commencèrent par attribuer toute nouvelle notion entrant dans leur champ cognitif et provenant du monde de la «civilisation ", toujours au même clan, celui des tatous (bokodori ecerae), mais rapidement, ce monde conceptuel nouveau prit le dessus au cœur même de leur système de pensée. Aujourd'hui, l'invasion de produits culturels de la civilisation technologique qui les entoure a pratiquement enseveli l'aroe, le rendant de moins en moins nécessaire. Le rôle du chamane des âmes devient peu à peu redondant et il ne lui incombe plus aujourd'hui que certaines fonctions, surtout cérémonielles.

La croissante virulence des bope exprimée par certains Bororo et remarquée par Viertler (1991: 192) est significative. Elle coïncide avec la désintégration de l'aroe et en constitue la contrepartie. Celui-ci peut encore être recréé et renouvelé par les rites funéraires tandis que les bope ne peuvent plus être contrôlés comme autrefois.

Dans la vie de tous les jours, la pensée traditionnelle ne semble plus avoir de sens; presque toutes les activités quotidiennes sont touchées d'une manière ou d'une autre par des influences de la société environnante, même les cérémonies préparatoires aux expéditions collectives de pêche et de chasse (elles sont de moins en moins collectives, et les poissons et le gibier se raréfient.

Par contre, si la communauté décide d'organiser un enterrement, cela implique un investissement moral suivi pendant des mois, un engagement de ressources considérable et un dévouement total de la part de tout le village lors de l'enterrement définitif. Tout ce qui touche à ces rites ne concerne que le monde des Bororo; ainsi peuvent-ils être eux mêmes, faire revivre l'aroe et, pour une fois, oublier les pressions du monde extérieur et regagner un élément essentiel de leur identité culturelle

L'importance que la mort et la célébration de ses rites a pris pour cette société ne peut pas être surestimée. Un épisode récent illustre l'intensité des passions que la valeur symbolique d'un défunt peut enflammer. Le meneur entendu dans l'enregistrement cité plus haut décéda en août 1991; c'était un homme de grand prestige, un des derniers à posséder une vaste connaissance des rituels et jouant le rôle de chamane des âmes. Son premier enterrement se réalisa dans le village de Garças où il résida la dernière partie de sa vie et où il exerçait ses fonc- 


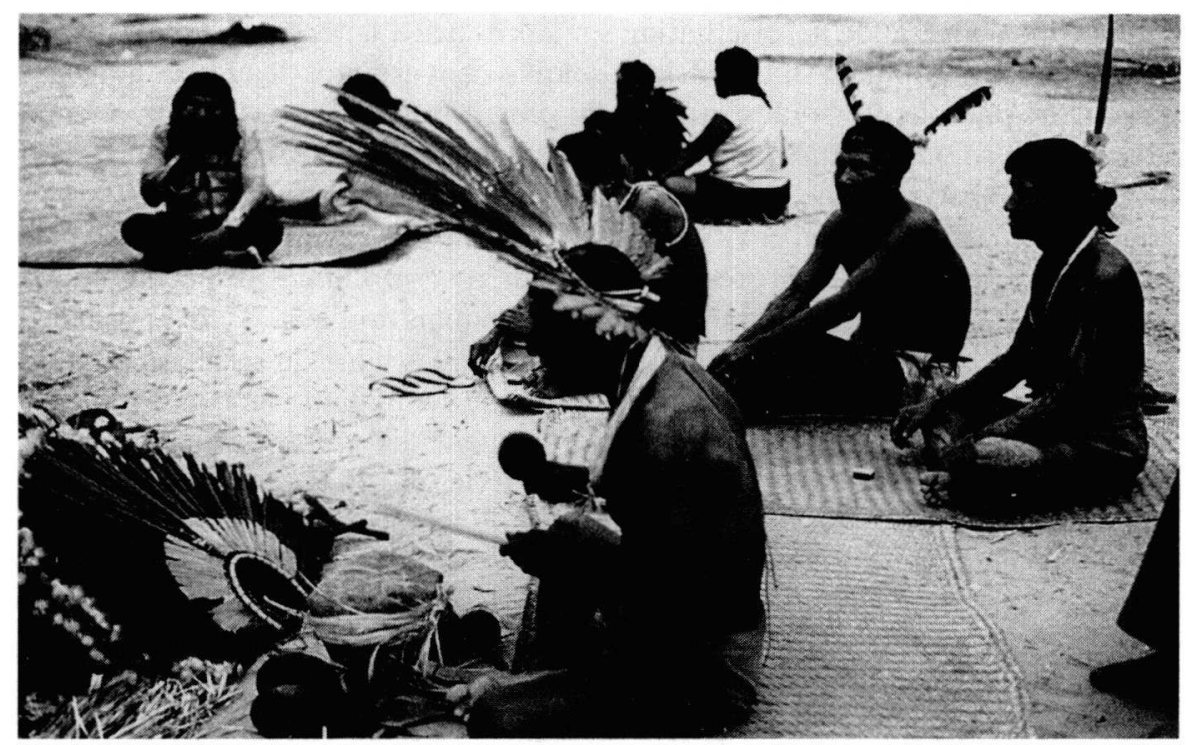

Fig. 5: Le chamane devant le tumulus s'accompagnant d'un petit hochet et du tambour $k a$. Photo: Priscilla Ellis.

tions rituelles. Une délégation d'un village d'une réserve voisine arriva pour réclamer le corps du mort, alléguant que les liens étroits du mort avec ce village où il avait résidé autrefois justifiait le transport du corps et la célébration des cérémonies finales à cet endroit. Des pourparlers s'ensuivirent, culminant dans un clair refus de la part des responsables de Garças. Les nouveaux venus ne lâchèrent pas prise et, prêts à tout pour obtenir la précieuse dépouille, revinrent au milieu de la nuit, armés; ils exhumèrent le corps et l'enlevèrent contre le gré de tout le village. Ils avaient dépensé de précieuses ressources pour obtenir un véhicule et tout le nécessaire pour transporter un cadavre putrescent à plusieurs centaines de kilomètres de là afin de célébrer pendant trois mois les jeux-représentations et de réaliser l'enterrement définitif.

Mais l'univers des aroe et les nouvelles formes de pensée permettant de faire face à la réalité qui les entoure et qui les encercle de plus en plus représentent deux mondes conceptuels irréconciliables pour qui a été élevé dans le système traditionnel bororo, dont les valeurs fondées sur le respect et le contact intime avec la nature et avec ses forces, ne parviennent pas à fournir un cadre de référence permettant aux Bororo de préserver, dans les circonstances actuelles, l'essentiel de leur identité.

De cette façon seule, la décision de respecter les rites funéraires donne aux Bororo la possibilité de survivre en tant que tels, les poussant en même temps à trouver refuge dans le dernier réduit qui leur est propre, la mort. 
On rencontre un état de désorientation similaire chez d'autres peuples autochtones dans les Amériques. Au Brésil, les quelques cent groupes de ce type qui existent encore ont tous été bouleversés par la colonisation, bien que leur expérience puisse varier selon la nature et la durée du contact. Pourrait-il se faire que la survie ou la disparition d'une ethnie dépende autant de sa capacité à revitaliser sa culture que des faits de son environnement? Si tel est le cas, et en tenant compte du double aspect du rôle des prescriptions pour la performance rituelle mentionnée plus haut (elles prescrivent un mode d'action mais font aussi vivre le système conceptuel), cette rigidité, qui est apparemment à l'origine de ce qu'on a interprété comme un manque d'adaptation du monde symbolique aux changements de l'environnement socio-culturel, porterait aussi les germes d'une possible résurrection (ou au moins les éléments de la survie) des Bororo comme groupe.

Le système conceptuel traditionnel doit rester largement intact, autrement le tout disparaît ; tant qu'il y aura des morts pour nourrir l'aroe, le respect des traditions rituelles funéraires est la seule voie ouverte aux Bororo pour les temps à venir. L'opportunité d'infuser leur culture avec vitalité et de pouvoir y restaurer un sens symbolique leur est ainsi donnée. Malgré la violence du front de colonisation, les épidémies et l'exploitation, le monde des esprits nourri par les rituels qui accompagnent la mort et le fascinant monde sonore qui lui appartient, ne seront-ils pas ce qui va permettre à l'identité des Bororo de perdurer? 


\section{ANNEXE}

Cette annexe a pour but de guider le lecteur lors de l'écoute de certains passages d'un disque de chant bororo (Canzio 1989) ${ }^{1}$ à l'aide d'une transcription. En présence d'enregistrements, le choix d'une méthode de transcription - nous l'avons dit ailleurs (Canzio 1991) - n'a plus comme autrefois le but de transmettre une image approximative de ce que l'auteur a entendu, mais surtout de faire ressortir les éléments de perception qui nous guideront vers une écoute aussi proche que possible de celle des membres de la culture concernée. Ce que nous avons tenté de réaliser vise précisement cet objectif et les notations qui suivent doivent être jugées selon ces critères.

Il faut relever ici qu'une notation conventionnelle sur portée ne nous rapprocherait pas de notre but car la pertinence de différents paramètres sonores dans l'exemple en question ne peut pas être facilement exprimée avec ces outils qui, d'ailleurs, connotent fortement d'autres types de musique. On remarquera aussi un écart plus ou moins marqué entre intention et réalisation. Nous avons décidé de représenter ici le normatif pour guider l'écoute et donner au lecteur des éléments de jugement sur l'exécution, tout en attirant son attention sur les détails de la performance et l'interaction entre les individus.

Etant donné l'étroite relation entre texte et réalisation, nous avons considéré l'entité texte/chant comme formant un tout et nous avons sélectionné, pour notre transcription, quelques paramètres qui permettront de mieux décrire ce complexe. Ce choix n'est pas arbitraire, mais correspond aux traits les plus saillants du système sonore qui constitue le chant et son accompagnement instrumental.

Le caractère répétitif et régulier des chants est un premier élément qui se profile clairement. Il existe une périodicité se traduisant par des cycles de pulsations qui reviennent régulièrement: elle est déterminée par la formule mélodique. Nous appelons métrique l'étude des systèmes de versification des textes mesurés et des éléments qui caractérisent une section significative du système sonore tels que la quantité syllabique d'un texte non mesuré. Nous appelons mélodique tout ce qui concerne les contours mélodiques et leurs relations avec le texte (métrique) et les cycles rythmiques (périodicité). Ces contours mélodiques présentent aussi une autre caractéristique: une rythmique interne qui, par rapport aux cycles, crée un système secondaire d'accentuation. Finalement, l'étude détaillée des formules rythmiques d'accompagnement instrumental et leur exécution constitue notre dernier paramètre d'analyse.

Naturellement, tous ces éléments agissent de façon imbriquée; nous n'explicitons ces niveaux d'analyse que pour faciliter la description. Dans ce qui suit, nous avons sélectionné quelques exemples de chant relativement simples et nous illustrerons graphiquement l'application de ces paramètres d'analyse et leur mode d'interaction.

Le texte en Bororo des quatre premières strophes de l'oieigo est le suivant (voir traduction à la page 84). Chaque partie sera recitée à trois reprises (Canzio 1989: plage 9 , index 1 et 2).

Le disque constitue une sélection parmi plus de trente heures d'enregistrements digitaux réalisés avec un Sony PCM-F1 et des microphones Sennheiser MD 421. Les lieux d'enregistrement étaient la case centrale du village (microphones attachés aux poutres près du poteau central) et la cour cérémonielle. Les enregistrements originaux font partie des archives personnelles de l'auteur et peuvent être consultés. Des droits versés par l'Unesco pour la publication du disque ont été reversés au village de Garças et des copies de ces enregistrements ont été envoyées aux répresentants de la communauté. 
[1] oieigo eko duiaga eko bokodoriware iga oieigo wararere taagaruia mamuiawuge eiaruru umuguia aiadudoge eimejera atugo padarogwarewu oiadowu boroio arirewu oiaga urugu rerudode kuwogorewu cegimejera bopaguduia cibae eimejera tuiagagejewu okwabijire

[2] upodoje tuguje toro ecewu botaruduia taboguru jaiobejewu tameriworo rukorewuge etoiaga urugu paduiagu turemoduia bopaguduia cibaegiri bakororo apodoje okwa bukoriri

[3] ema tawiria uiroga purewore kwogorewu bopaguduia iturawore eimejera awiria uiroga ecewu ecera bororo bukejewuge eimejera atogewu ipareceba bakororo biria orewuwore (awuko)

[4] mamurewuge tagirenono mamarewuge tagiarega tare areaku kuiada eimejera ewoiga aio kwogorewu akara rekoducedo mamarewuge tamuguia iturabo wuiajetugae $^{2}$

Il s'agit ici de textes non mesurés auxquels on appliquera une formule de récitation simple. L'application de cette formule au texte est intimement liée à certains aspects de la prosodie bororo que nous aborderons ci après.

La structure phonotactique de la syllabe bororo est consonne optionelle plus voyelle; soit selon les conventions de la linguistique: $\mathrm{S}=(\mathrm{C}) \mathrm{V}$. L'accent tonique principal ou stress dans le mot bororo tombe toujours sur l'avant dernière syllabe, il existe des accents toniques secondaires à chaque deuxième syllabe précédente. Ainsi, si un mot contient deux syllabes l'accent sera situé sur la première; si le mot contient trois syllabes l'accent tombera sur la deuxième; s'il en comporte quatre, sur la troisième et la première syllabe et ainsi de suite. La formule, dans notre cas, s'étend sur deux syllabes. Nous trouvons donc une relation entre accent tonique et nombre de syllabes: mot à nombre de syllabes paires, accents sur les syllabes impaires, et mot à nombre de syllabes impaires, accents sur les syllabes paires.

La formule de récitation appliquée aux deux premières strophes de l'oieigo fractionne le texte en groupes de deux syllabes et modifie le texte, selon le cas, de la façon suivante: 1) si le mot a un nombre pair de syllabes, la formule s'étendra chaque fois sur deux syllabes tout en répétant la voyelle de la dernière syllabe deux fois. Ainsi, nous avons quatre unités syllabiques égales débitées au rythme d'une par pulsation;2) si le mot possède un nombre impair de syllabes, à la première syllabe on ajoutera sa voyelle répétée trois fois et nous aurons à nouveau ici quatre unités syllabiques. Par exemple, cette formule appliquée au mot oieigo fractionné comme suit: oie/igo donnera o.ie.e.e i.go.o.o et appliquée au mot duiaga fractionné comme suit: du/iaga donnera du.u.u.u ia.ga.a.a. ${ }^{3}$

Si le mot commence par une syllabe accentuée (donc un mot possédant un nombre pair de syllabes) la première variante s'applique à chaque groupe de deux syllabes; si le mot commence par une syllabe non accentuée (donc un mot à nombre impair de syllabes)

2 Nous adoptons ici une orthographe simplifiée adaptée de Crowell (1979) et non le système complexe et plus détaillé utilisé par Albisetti et Venturelli.

3 Sans entrer dans les détails de la phonémique bororo, nous devons dire qu'il ne faut pas confondre orthographe avec forme phonétique, car l'ortographe adoptée suit plutôt la prononciation de la chaîne parlée que celle de textes chantés. Ainsi $i$ et $u$, selon le contexte peuvent devenir phonétiquement de demi-voyelles et donc fonctionner comme des consonnes. Par exemple duiaga et kuogoreu, se séparent en du·ia·ga et ku·wo·go·re·wu. 
la deuxième variante est appliquée à la première syllabe et la première variante à chaque groupe de deux syllabes dans le mot. Cela pourrait être exprimé comme suit: pour un groupe de deux syllabes: $S_{1} S_{2} V_{2} V_{2}$; pour un groupe monosyllabique: $S_{1} V_{1} V_{1} V_{1}$. Les instances de fractionnement monosyllabique sont indiquées en italiques.

Voyons d'abord le fractionnement textuel, puis la forme que prend le texte une fois modifié par la formule appliquée aux deux premières strophes.

Fractionnement textuel (normatif):

[1] oie/igo eko du/iaga eko boko/dori/ware iga oie/igo wara/rere (ta/aga/ruia) ma/muia/wuge eia/ruru umu/guia aia/dugo/doge e/ime/jera a/tugo pada/rogwa/rewu oia/dowu bo/roio ari/rewu o/iaga u/rugu reru/dode $\mathrm{ku} / \mathrm{wogo} / \mathrm{rewu} \mathrm{ce} /$ gime/jera bo/pagu/duia ci/bae e/ime/jera tuia/gaje/jewu $\mathrm{o} / \mathrm{kwabi} / \mathrm{jire}$

[2] upo/doje tu/guje toro e/cewu bo/taru/duia tabo/guru ja/iobe/jewu ta/meri/woro ru/kore/wuge eto/iaga u/rugu padu/iagu tu/remo/duia bo/pagu/duia ci/baie/giri bako/roro apo/doje okwa buko/riri

Réalisation:

[1] o.ie.e.e i.go.o.o e.ko.o.o du.u.u.u ia.ga.a.a e.ko.o.o bo.ko.o.o do.ri.i.i wa.re.e.e i.ga.a.a o.ie.e.e i.go.o.o wa.ra.a.a re.re.e.e ta.a.a.a ga.ru.u.u wu.ia.a.a (au lieu de a.ga.a.a ru.ia.a.a) ma.a.a.a mu.ia.a.a wu.ge.e.e e.ia.a.a ru.ru.u.u u.mu.u.u. e.e.e.e i.me.e.e je.ra.a.a a.a.a.a tu.go.o.o pa.da.a.a ro.gwa.a.a re.wu.u.u o.ia.a.a do.wu.u.u bo.o.o.o ro.io.o.o a.ri.i.i re.wu.u.u o.o.o.o ia.ga.a.a u.u.u.u ru.gu.u.u re.ru.u.u do.de.e.e $k u . u . u . u$ wo.go.o.o re.wu.u.u ce.e.e.e gi.me.e.e je.ra.a.a bo.o.o.o pa.gu.u.u du.ia.a.a ci.i.i.i ba.ie.e.e e.e.e.e i.me.e.e. je.ra.a.a tu.ia.a.a ga.je.e.e je.wu.u.u o.o.o.o kwa.bi.i.i ji.re.e.e (awuko)

[2] u.po.o.o do.je.e.e tu.u.u.u gu.je.e.e to.ro.o.o e.e.e.e ce.wu.u.u bo.o.o.o ta.ru.u.u du.ia.a.a ta.bo.o.o gu.ru.u.u ja.a.a.a io.be.e.e je.wu.u.u ta.a.a.a me.ri.i.i wo.ro.o.o ru.u.u.u ko.re.e.e wu.ge.e.e e.to.o.o ia.ga.a.a u.u.u.u ru.gu.u.u pa.du.u.u ia.gu.u.u tu.u.u.u re.mo.o.o du.ia.a.a bo.o.o.o pa.gu.u.u du.ia.a.a ci.i.i.i ba.ie.e.e gi.ri.i.i ba.ko.o.o ro.ro.o.o a.po.o.o do.je.e.e o.kwa.a.a bu.ko.o.o ri.ri.i.i (o toiogire) (op. cit.: index $3 /$ temps $02: 28$ )

Ainsi, nous avons deux variantes de la même formule, conditionnées par le contexte phonémique du texte: le fractionnement textuel est fonction de l'accent tonique. La formule impose toujours la périodicité (quatre pulsations dans notre cas) et leur rythme interne (une syllabe par pulsation). Le contour mélodique est ici nul car il n'y a pas de hauteurs contrastantes. La figure 1 montre cela schématiquement.

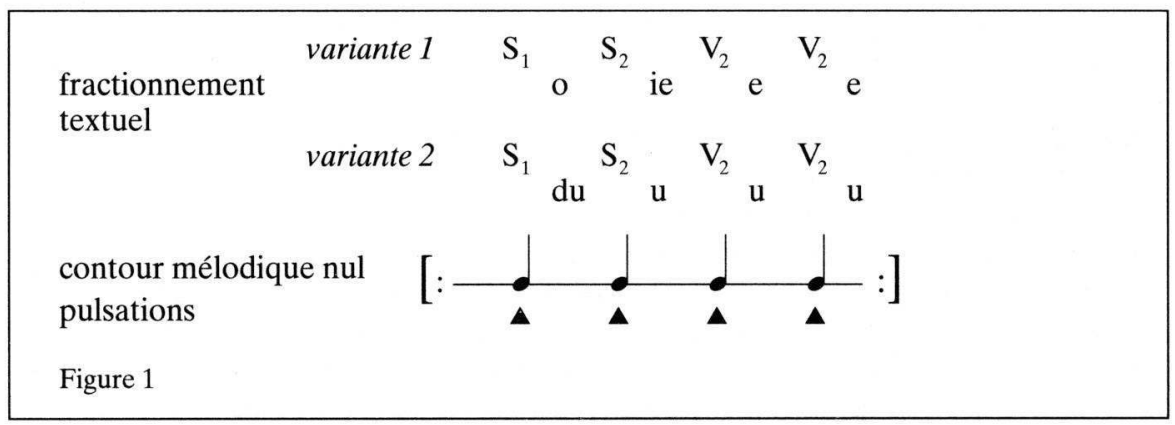


L'accompagnement instrumental pose un problème de description lié à la technique particulière de jeu des hochets. Une simple transcription auditive ne pourra pas faire ressortir les éléments significatifs de l'accompagnement et ne nous permet pas une formalisation pertinente de la formule; ce sont les timbres obtenus avec des mouvements particuliers qui comptent. C'est à cause de cela que nous avons opté pour un type de transcription que nous appelons articulatoire, par analogie à la terminologie utilisée en phonétique. Elle consiste en une description des gestes employés pour produire les sons.

Une paire de petits hochets (bapo rogu) est employée par le meneur pour rendre les formules rythmiques instrumentales qu'accompagnent les exemples presentés ici. Deux mouvements de base sont utilisés: 1) la main gauche bat régulièrement la pulsation avec un coup descendant sec et appuyé, 2) la main droite effectue une brusque descente produisant un son chuintant suivi d'un coup de poignet vers l'extérieur qui laisse entendre une attaque claire. Nous représentons cela comme suit:

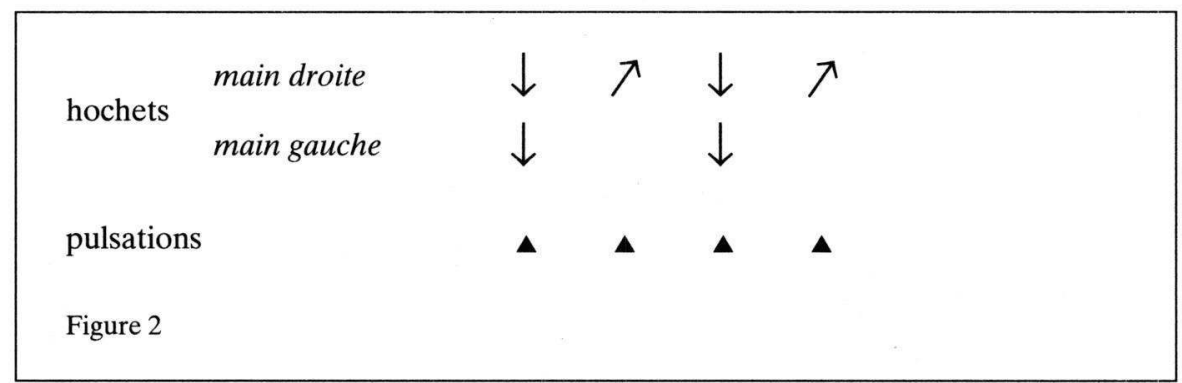

La formule utilisée pour chanter les troisième et quatrième strophes fractionne le texte de la même façon que la formule précédente et s'étend aussi sur quatre pulsations, mais avec une rythmique interne différente (Fig. 3) et un contour mélodique fondé sur l'opposition de deux hauteurs constrastantes, et une hauteur intermédiaire fonctionnant comme note de passage toujours montante entre les deux autres. Nous avons ici deux variantes définies par leur contour mélodique; leur application dépend, comme dans le premier cas, de la présence (variante 1) ou de l'absence (variante 2) d'accent tonique sur la syllabe qui commence le mot. Toutes les instances de la variante 2 sont indiquées en italiques.

En écoutant les strophes [3] et [4] (op. cit.: plage 9, index 17 et 18) nous notons que le fractionnement textuel, ne correspond pas toujours à ce que notre hypothèse de travail prévoit; notamment les instances marquées en gras. Nous expliquons ces anomalies comme étant des erreurs de performance. En effet, d'autres enregistrements du même oieigo en notre possession le prouvent. Ce défaut d'accord entre le prescriptif et sa réalisation est un phénomène que nous avons observé à maintes reprises, particulièrement dans les chants à texte mesuré. Nous essayerons ensuite d'expliquer en détail le type d'interaction entre le meneur et l'assemblée des hommes, les départs du normatif et enfin le type d'action corrective appliqué.

En ce qui concerne la première instance de segmentation incorrecte du texte (index 17/temps 39:17); nous notons qu'il y a un chanteur au premier plan qui se détache du reste du chœur; il se trompe en chantant wore qui devrait être wo.re.e.e avec le contour mélodique de la variante 1. Il fractionne mal le texte, chante wo.o.o.o en appliquant la variante 2 et re.e.e.e avec la variante 1. A ce moment le meneur (la voix basse qui anticipe le texte et son fractionnement), intervient et souffle le fractionement correct du 
texte qui suit. Les deux instances suivantes de découpage erroné du texte rélèvent d'une méprise similaire; en orewuwore il y a une méprise non corrigée et en tamuguia le chanteur applique la variante 2 à la première syllabe d'un mot accentué, mais le meneur intervient immédiatement en soufflant la syllabe manquante et le groupe suivant de syllabes au premier découpage.

Les mots awuko = écoutez, et o toiogire = se termine! sont des termes introduits respectivement pour marquer la transition de la première à la deuxième partie et pour indiquer la fin de la récitation.

Fractionnement textuel (normatif):

3] e/ma tawi/ria ui/roga $\mathrm{pu} / \mathrm{rewu} /$ wore $\mathrm{ku} /$ wogo/rewu bo/pagu/duia $\mathrm{i} /$ ture/wore e/ime/jera awi/ria ui/roga e/cewu e/cera bo/roro bu/keje/wuge $\mathrm{e} / \mathrm{ime} / \mathrm{jera}$ ato/gewu i/pare/ceba bako/roro bi/ria o/rewu/wore

4] $\mathrm{ma} /$ mure/wuge ta/gire/nono ma/mare/wuge ta/gia/rega tare/aku $\mathrm{ku} /$ iada e/ime/jera ewo/iga aio ku/wogo/rewu a/karu re/kodu/cedo ma/mare/wuge $\mathrm{tamu} /$ guia itu/rabo wuia/jetu/gaie

\section{Réalisation:}

3] e.ma.a.a ta.wi.i.i ri.a.a.a ui.i.i.i ro.ga.a.a pu.u.u.u re.wu.u.u wo.o.o.o re.e.e.e (au lieu de wo.re.e.e) $k u . u . u . u$ wo.go.o.o re.wu.u.u bo.o.o.o pa.gu.u.u du.ia.a.a i.i.i.i tu.re.e.e wo.re.e.e e.e.e.e i.me.e.e je.ra.a.a a.wi.i.i ri.ia.a.a u.i.i.i ro.ga.a.a e.e.e.e ce.wu.u.u e.e.e.e ce.ra.a.a bo.o.o.o ro.ro.o.o bu.u.u.u ke.je.e.e wu.ge.e.e e.e.e.e i.me.e.e je.ra.a.a to.o.o.o ge.wu.u.u i.i.i.i pa.re.e.e ce.ba.a.a ba.ko.o.o ro.ro.o.o bi.i.i.i ri.ia.a.a o.o.0.0 re.wu.u.u (au lieu de o.o.o.o re.wu.u.u) wo.re.e.e (awuko)

4] ma.a.a.a mu.re.e.e wu.ge.e.e ta.a.a.a gi.re.e.e no.no.o.o ma.a.a.a ma.re.e.e wu.ge.e.e ta.a.a.a gi.ia.a.a re.ga.a.a ta.re.e.e a.ku.u.u ku.u.u.u ia.da.a.a e.e.e.e i.me.e.e je.ra.a.a e.wo.o.o i.ga.a.a a.io.o.o ku.u.u.u wo.go.o.o re.wu.u.u a.a.a.a ka.ru.u.u re.e.e.e ko.du.u.u ce.do.o.o ma.a.a.a mu.re.e.e wu.ge.e.e ta.a.a.a (au lieu de ta.mu.u.u le meneur dit: muguia) gu.ia.a.a i.tu.u.u ra.bo.o.o wu.ia.a.a je.tu.u.u ga.ie.e.e

Les transcriptions qui suivent (Figs. 4 et 5) montrent les paramètres d'analyse pour les deux contours mélodiques.

fractionnement

textuel variante 1

contour mélodique

pulsations

hochets

main droite

main gauche

\section{$\begin{array}{llll}\mathrm{S}_{1} & \mathrm{~S}_{2} & \mathrm{~V}_{2} & \mathrm{~V}_{2}\end{array}$}

e ma a

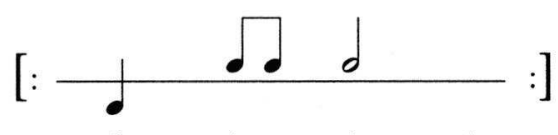

ム $\Delta$

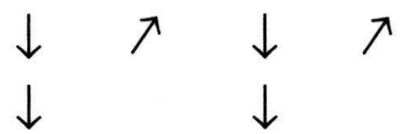

Figure 4 
fractionnement

textuel variante 2

contour mélodique

pulsations

main droite

main gauche

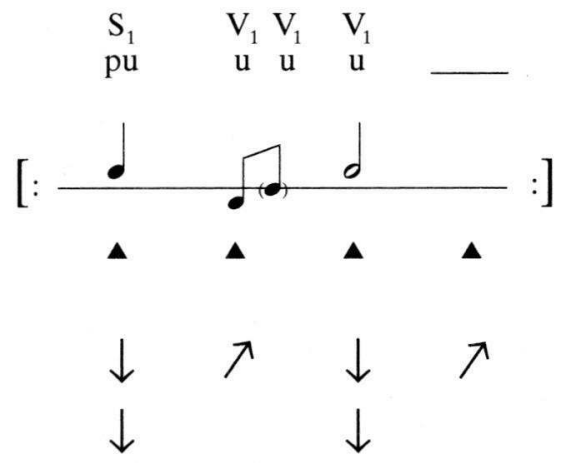

Figure 5

\section{Bibliographie}

ALBISETTI César et VENTURELLI Ângelo

1962 Enciclopédia Bororo, Vol 1. Campo Grande: Muséu Regional Dom Bosco.

1969 Enciclopédia Bororo, Vol 2. Campo Grande: Muséu Regional Dom Bosco.

1976 Enciclopédia Bororo, Vol 3. Campo Grande: Muséu Regional Dom Bosco.

AYTAI Desidério

1985 O mundo sonoro Xavante. São Paulo: Universidade de São Paulo. Coleção Museu Paulista: Etnologia vol 5.

BORDIGNON Mario Enawuréu

1987 Os Bororo na Historia do Centro Oeste Brasileiro 1716-1986. Campo Grande: Missão salesiana do Mato Grosso.

CANZIO Riccardo

1989 Le monde sonore des Bororo. CD Unesco D 8201. Paris: Auvidis.

1991 Compte rendu de Why Suyá sing. A musical anthropology of an Amazonian people de A. Seeger. Cahiers de musiques traditionnelles (Genève) 4: 268-273.

COLBACCHINI Antonio

1925 I Bororos Orientali «Orarimugudoge» del Mato Grosso. Torino: Contributi Scientifici delle Missioni Salesiane del Venerabile Don Bosco, vol I.

CROCKER Jon Christopher

1985 Vital Souls: Bororo Cosmology, Natural Symbolism and Shamanism. Tucson: University of Arizona Press.

CROWELL Thomas Harris

1979 A Grammar of Bororo. PhD thesis. Cornell University.

GEERTZ Clifford

1973 The Interpretation of Cultures: Selected Essays. New York: Basic Books. 
LEVI-STRAUSS Claude

1955 Tristes Tropiques. Paris: Plon.

1958 Anthropologie structurelle. Paris: PIon.

MERRIAM Alan Parkhurst

1960 Ethnomusicology: discussion and definition of the field. Ethnomusicology 4:107-114.

\section{SEEGER Anthony}

1980 «Sing for your sister. The Structure and Performance of Suyá akia». In: McLeod, Norma et Herndon, Marcia (eds). The Ethnography of Musical Performance. Norwood, PA: Norwood Editions.

1987 Why Suyá sing. Cambridge University Press.

VIERTLER Renate

1991 A refeição das almas. Uma interpretação etnológica do funeral dos índios Bororo. São Paulo: HUCITEC: Editora da Universidade de São Paulo. 